\title{
Predicting cerebrospinal fluid leakage prior to posterior circumferential decompression for the ossification of the posterior longitudinal ligament in the thoracic spine
}

\author{
Jun Zhong, Bingtao Wen, Zhongqiang Chen \\ Department of Orthopedic, Peking University International Hospital, Beijing, China \\ Contributions: (I) Conception and design: J Zhong, B Wen; (II) Administrative support: B Wen; (III) Provision of study materials or patients: J Zhong, \\ Z Chen; (IV) Collection and assembly of data: J Zhong, Z Chen; (V) Data analysis and interpretation: All authors ; (VI) Manuscript writing: All \\ authors; (VII) Final approval of manuscript: All authors. \\ Correspondence to: Bingtao Wen. Department of Orthopedic, Peking University International Hospital, No. 1 Life Science Park Road, Zhongguancun \\ Life Science Park, Changping District, Beijing 102206, China. Email: bingtaowen_binter@126.com.
}

\begin{abstract}
Backgrounda Cerebrospinal fluid leakage (CSFL) is one of the most common complications after posterior transarticular osteotomy and circumferential decompression (CD) for the ossification of the posterior longitudinal ligament (OPLL) in the thoracic spine. Predicting CSFL preoperatively would be of great value to surgeons. The aim of this study was to discover the factors that can predict CSFL prior to CD and incorporate them into a predictive model.
\end{abstract}

Methods: Sixty-one patients with OPLL who underwent posterior transarticular osteotomy and CD at Peking University International Hospital were divided into a CSFL group and a non-CSFL group. Univariate analysis was used to identify possible predictors. A multivariate logistic regression model was developed to predict the probability of CSFL. Model validation was performed using a receiver operating characteristic (ROC) curve.

Results: CSFL occurred in $31.1 \%$ of patients. Univariate regression analysis showed statistical differences $(\mathrm{P}<0.05)$ in smoking history, segment of $\mathrm{CD}$, whether OPLL was combined with the ossification of the ligamentum flavum (OLF), number of laminectomies, occupying ratio, and OPLL base ratio. Our multivariate regression model showed that CSFL predictors included smoking history [odds ratio (OR) $=30.1 ; \mathrm{P}=0.003$ ], the upper thoracic segment $(\mathrm{OR}=188.0 ; \mathrm{P}=0.002)$, the middle thoracic segment $(\mathrm{OR}=57.4$; $\mathrm{P}=0.005)$, and the OPLL base ratio $(\mathrm{OR}=1.3 ; \mathrm{P}=0.007)$. The $\mathrm{ROC}$ curve was in the upper left corner [area under the curve $=0.955,95 \%$ confidence interval $(\mathrm{CI}): 0.91-1.00 ; \mathrm{P}<0.001]$, indicating the model had good predictability.

Conclusions: The predictive model shows that if patients with thoracic OPLL have a history of smoking, or the segment of CD is in the upper or middle thoracic spine, or the OPLL has a wide base, The possibility for postoperative CSFL occurring is higher. Using these factors, a surgeon can preoperatively warn patients of the probability of CSFL occurring after posterior transarticular osteotomy and CD.

Keywords: Cerebrospinal fluid leakage (CSFL); circumferential decompression (CD); thoracic ossification of posterior longitudinal ligament; predictive model

Submitted Jul 23, 2021. Accepted for publication Oct 16, 2021.

doi: 10.21037/apm-21-2323

View this article at: https://dx.doi.org/10.21037/apm-21-2323

(c) Annals of Palliative Medicine. All rights reserved. 


\section{Introduction}

The occurrence of ossification of the posterior longitudinal ligament (OPLL) in the thoracic spine is about $0.8 \%(1)$, which is lower than that of OPLL in the cervical spine. However, due to the limited activity of thoracic spine as well as poor compensatory effect of thoracic spinal cord, bone compression often requires static compression $(2,3)$. Therefore, once clinical symptoms of OPLL occur in the thoracic spine, they progress rapidly and can cause severe spinal cord injury or even paraplegia in a short time. Surgery is the only way to treat OPLL in the thoracic spine.

Simple thoracic laminectomy used to be a safe surgical method. However, this technique could hardly obtain sufficient decompression for patients with severe ventral spinal cord compression, ossification of the beak posterior longitudinal ligament or severe kyphosis. Circumferential decompression (CD) has been widely used due to its effectiveness in removing the compression around the spinal cord even postoperative complication rate was relative higher. Many scholars have confirmed that the posterior approach can achieve satisfactory surgical results $(2,3)$. Nevertheless, the operation is highly difficult and can result in many complications, with cerebrospinal fluid leakage (CSFL) being one of the most common (4). CSFL increases the risk of postoperative infection (5), can lead to the infection of the central nervous system, and result in the long-term formation of cerebrospinal fluid pseudocysts (6). At present, dural ossification is considered to be the main cause of intraoperative dural injury. However, neither computed tomography (CT) nor magnetic resonance imaging (MRI) can determine dural ossification in advance. In addition, these radiologic changes could hardly represent spinal cord compression, which means that dural ossification is often not confirmed until the operation is underway.

For this reason, our study examined which preoperative factors can help predict CSFL and thereby be used to inform surgeons in advising patients of their individual CSFL risk prior to surgery. Accordingly, the aim of this study was to identify those preoperative factors that can predict CSFL and use them to establish a predictive model.

We present the following article in accordance with the STARD reporting checklist (available at https://dx.doi. org/10.21037/apm-21-2323).

\section{Methods}

\section{Patient screening and selection}

The patients enrolled in this study were diagnosed with OPLL in the thoracic spine between August 2015 and June 2020 at Peking University International Hospital. The patient inclusion criteria were as follows: (I) the patient was $\geq 18$ years old, (II) the surgery plan included a simple posterior approach to transarticular osteotomy and $\mathrm{CD}$, (III) patients underwent complete preoperative CT examination to determine the type of OPLL and whether there was ossification of the ligamentum flavum (OLF), and (IV) surgery was performed by the same team. The exclusion criteria included (I) patient age $<18$ years old, (II) patients with congenital or acquired deformities in the thoracic spine (e.g., congenital thoracic scoliosis, ankylosing spondylitis, and kyphosis secondary to spinal tuberculosis), (III) thoracic infectious diseases, (IV) primary or metastatic spinal tumors, and $(\mathrm{V})$ previous history of thoracic surgery. According to the inclusion and exclusion criteria, a total of 61 patients were included in the study. Among them, 29 were males and 32 were females. The age range was 27 82 years $(53.4 \pm 13.0$ years), and the duration of treatment (from clinical symptoms to surgery) was $1-36$ months (9.2 \pm 8.6 months).

All procedures performed in this study involving human participants were in accordance with the Declaration of Helsinki (as revised in 2013). The study was approved by ethics board of Peking University International Hospital [No. 2021-006(BMR)] and informed consent was taken from all the patients.

\section{Surgical procedure and treatment of dural defect}

All patients underwent transarticular osteotomy and CD. Firstly, laminectomy was used to complete the dorsal decompression, and the bilateral articular process was removed. We then performed the oblique forward inward resection of the OPLL anterior intervertebral disk. According to the scope of the upper and lower OPLL, part of the posterior vertebrae bone or even part of the pedicle was removed if it was necessary to "penetrate" on both sides. Finally, the OPLL was pushed forward to the intervertebral 
space, and the OPLL block was removed from the back side to complete the decompression of the ventral spinal cord.

Dural defect often occurred in two steps of the operation: either when the laminectomy was performed or when the OPLL block was pushed into the intervertebral space so that it could be separated from the dural mater. Dural defects may also occur if a serious adhesion between the ossification block and dural mater was found. In this study, ventral dural injury or defects were generally not repaired due to surgical field limitations. Usually, dorsal dural injury was first treated with a direct suture with a 5-0 vessel suture, and if difficult sutures or large dural defects occurred, brain cotton was temporarily used to cover the suture to ensure a clear field. After spinal cord decompression, a whole piece of gelatin sponge was used to cover the dural injury without repair of large dural defects. The surgical incision was then closed layer by layer by strict continuous suture.

\section{Diagnostic criteria for CSFL}

Patients were diagnosed with CSFL if their symptoms met any of the following diagnostic criteria: (I) a dural rupture or defect was found during the operation, or cerebrospinal fluid outflow was seen; (II) large amounts ( $>300 \mathrm{~mL} /$ day) of clear or light blood drainage fluid outflow at the wound drainage tube remained visible for at least 48 hours after surgery; and (III) the patients developed severe headaches, nausea, vomiting, or other low cranial pressure symptoms.

\section{Postoperative management of CSFL}

Once a patient was found to have CSFL, a unified treatment process was adopted. This included (I) ensuring the patient was in a continuous lateral position without a pillow, replacing the wound negative pressure drainage ball by atmospheric drainage bag between 12 and 24 hours after surgery, and placing the atmospheric drainage bag at the same horizontal position of the surgical incision. (II) If the patient had symptoms of low intracranial pressure such as headache, the bed tail would be raised $10 \mathrm{~cm}$, or the Trendelenburg position would be adopted to alleviate the symptoms. (III) Water and electrolytes were administered on a regular basis to strengthen nutritional support. For patients with low intracranial pressure symptoms, intravenous infusion of concentrated sodium chloride was conducted to alleviate the symptoms. (IV) The patient was made aware of the infection, and the use of antibiotics was extended accordingly. The use of antibiotics ceased after the drainage tube was removed and the patient's body temperature returned to normal for 3 consecutive days. (V) The wound drainage tube was kept for at least 5 days but generally not more than 1 week. After the color of the drainage fluid was basically clear, the drainage tube could be removed. Finally, 1 to 2 stitches were then sutured in the whole layer of the drainage tube to avoid the outflow of cerebrospinal fluid from the drainage port.

\section{Predictor of CSFL}

Patients were divided into a CSFL group and a nonCSFL group based on the CSFL diagnostic criteria. The predictors we considered were the following: (I) demographic characteristics, including age, gender, and body mass index (BMI); (II) clinical data, including patient's preoperative Japanese Orthopaedic Association (JOA) score, course of disease, complications (hypertension, diabetes), smoking history, segment of CD (upper, middle, lower thoracic segments), and the number of laminectomy segments; and (III) radiological data, including preoperative thoracic CT and MRI that were performed in all enrolled cases before surgery. According to the thoracic CT sagittal image, the OPLL was divided into three types: isolated, continuous (flat or wavy), and hybrid. The medical personnel would determine whether the segment of CD was combined with OLF. The occupying ratio was defined as the OPLL thickness divided by the front-and-rear diameter of the spinal canal, which was measured on the sagittal CT scan. The OPLL base ratio was defined as the OPLL width divided by the transverse diameter of the spinal canal, which was measured on the axial CT scan (Figure 1). The intramedullary high signal was defined in the thoracic MRI T2-weighted image.

\section{Statistical analysis}

This was a retrospective cohort study, and SPSS 19.0 statistical software (IBM Corporation, Armonk, NY, USA) was used. The measurement data are expressed with the mean \pm standard deviation. Single-factor regression analysis was used to analyze whether possible predictors had statistical significance. Predictors that were of statistical significance were included in the multivariate regression analysis. A predictive model was constructed, and a receiver operating characteristic (ROC) curve was drawn. 


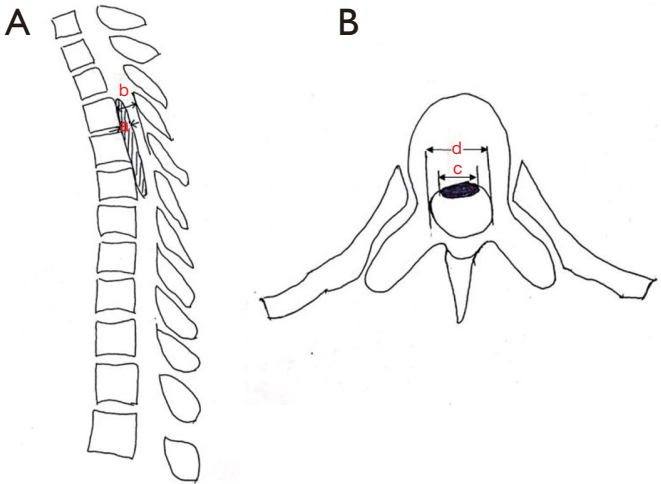

Figure 1 Measurement methods for the occupying ratio and OPLL base ratio. (A) The occupying ratio was defined as the OPLL thickness divided by the front-and-rear diameter of the spinal canal, which was measured on the CT scan $(\mathrm{a} / \mathrm{b} \times 100 \%)$; (B) the OPLL base ratio was defined as the OPLL width divided by the transverse diameter of the spinal canal on the axial CT scan $(\mathrm{c} /$ $\mathrm{d} \times 100 \%$ ). OPLL, ossification of posterior longitudinal ligament.

\section{Results}

\section{Incidence of CSFL}

According to our CSFL diagnostic criteria, 19 patients were included in the CSFL group and 42 patients were included in the non-CSFL group. The incidence of CSFL among the patients was $31.1 \%$. Among the CSFL group, 7 were males and 12 were females, with an average age of $49.8 \pm 11.4$ years. Dural injury was found in 14 of these 19 cases, including 10 with a ventral dural injury, and 4 with a dorsal dural injury. Although no dural injury was found in the other 5 cases, they met the diagnostic criteria of CSFL (Table 1).

\section{Complications related to CSFL}

Of the 19 CSFL patients, 12 cases (63.2\%) had symptoms of low intracranial pressure, such as headache, and the symptoms were relieved after the removal of the drainage tube; 1 patient $(5.3 \%)$ had cerebrospinal fluid outflow from the incision, and the wound healed successfully after debridement; and 1 case $(5.3 \%)$ was readmitted to the hospital for fever 3 weeks after the operation, with the wound effusion observably cured after 2 weeks of antiinfective treatment. No patient had complications such as falling pneumonia, urinary tract infection, central nervous system infection, or venous thrombosis of the lower extremity. The average retention time of the drainage tube was 5.6 \pm 1.0 days, which was significantly higher than that of the non-CSFL group $(4.2 \pm 1.3$ days; $\mathrm{P}<0.001)$. The average hospitalization days in the CSFL group were $16.3 \pm 6.3$ days, which was slightly higher than that in the non-CSFL group (15.8 \pm 6.7 days), but there was no statistical difference $(\mathrm{P}=0.77)$.

\section{Predictor of CSFL}

Univariate regression analysis showed statistical differences $(\mathrm{P}<0.05)$ in smoking history, segment of $\mathrm{CD}$, whether OPLL was combined with OLF, number of laminectomies, occupying ratio, and OPLL base ratio (Table 2). The above variables were included in the multivariate regression model, and were entered in the way of "step forward (condition)" when the statistical software was run. The resulting logistic model was statistically significant $\left(\chi^{2}=44.78 ; \mathrm{P}<0.001\right)$. The model correctly classified $86.9 \%$ of the research objects, with a sensitivity of $73.7 \%$ and a specificity of $90.5 \%$. Among the 6 variables mentioned above, smoking history, segment of CD, and OPLL base ratio were statistically significant (Table 3). The regression equation was as follows:

$\mathrm{Ln}$ (odds) $=-20.09+3.41 \times \mathrm{S}+5.24 \times \mathrm{U}+4.05 \times \mathrm{M}+0.22 \times \mathrm{B}$

where $S$ represents smoking $(0=$ no, $1=$ yes $), U$ represents upper thoracic $(0=$ no, $1=$ yes), $M$ represents middle thoracic $(0=$ no, $1=$ yes $)$, and $B$ represents base ratio.

A Hosmer-Lemeshow test was used to analyze the fitting degree of the model. The results showed that the difference was not statistically significant $(\mathrm{P}=0.62)$, indicating that the model fitted well and could be used for risk prediction. The Predicted probability was used as the target variable and the CSFL as the state variable, and a ROC curve was drawn. It was shown that the ROC curve was in the upper left corner [area under the curve $=0.955 ; 95 \%$ confidence interval (CI): 0.91-1.00; $\mathrm{P}<0.001]$, indicating the model had good predictability (Figure 2). The maximum Youden index (Youden index $=$ sensitivity + specificity -1 ) of this model was 0.81 . This model had a sensitivity of $94.7 \%$ and a specificity of $85.7 \%$. A typical case was shown in Figure 3.

\section{Discussion}

CSFL is a common complication resulting from spinal surgery, and the sites at which it can occur vary greatly. Hannallah et al. (7) reported an incidence of CSFL after cervical surgery of only $1 \%$, while Galarza et al. (8) reported an incidence of CSFL after lumbar vertebrae surgery of $3.2 \%$. However, the incidence of CSFL after thoracic surgery has been found to be much higher than 
Table 1 The comparison of demographic characteristics and radiological and surgical data between the CSFL group and non-CSFL group

\begin{tabular}{|c|c|c|c|}
\hline Variable & CSFL group & Non-CSFL group & $P$ value \\
\hline Age & $49.8 \pm 11.4$ & $55.0 \pm 13.5$ & 0.15 \\
\hline BMI $\left(\mathrm{kg} / \mathrm{m}^{2}\right)$ & $27.6 \pm 4.5$ & $28.0 \pm 5.4$ & 0.81 \\
\hline Duration of disease (month) & $11.2 \pm 10.2$ & $8.3 \pm 7.7$ & 0.22 \\
\hline Diabetes mellitus (yes/no) & $1 / 18$ & $3 / 39$ & 1.00 \\
\hline Hypertension (yes/no) & $5 / 14$ & $7 / 35$ & 0.49 \\
\hline Smoking (yes/no) & $16 / 3$ & $13 / 29$ & $<0.001$ \\
\hline Segment of CD & & & 0.001 \\
\hline Lower & 2 & 24 & \\
\hline Type of OPLL & & & 0.21 \\
\hline Beaked & 8 & 25 & \\
\hline Non-beaked & 11 & 17 & \\
\hline Combined with OLF (yes/no) & $13 / 6$ & $13 / 29$ & 0.006 \\
\hline Occupying ratio (\%) & $43.4 \pm 7.6$ & $36.1 \pm 6.8$ & $<0.001$ \\
\hline OPLL base ratio (\%) & $65.2 \pm 6.2$ & $56.9 \pm 7.9$ & $<0.001$ \\
\hline Intramedullary high signal (yes/no) & $3 / 16$ & $6 / 36$ & \\
\hline
\end{tabular}

CSFL, cerebrospinal fluid leakage; BMI, body mass index; JOA, Japanese Orthopaedic Association; CD, circumferential decompression; OPLL, ossification of posterior longitudinal ligament; OLF, ossification of the ligamentum flavum.

that of other parts of the spine, which is reported to be about $20-40 \%$ (9-11). Moreover, the operative approach required for different areas differs significantly. From 362 cases of thoracic surgery, $\mathrm{Hu}$ et al. (12) found the overall incidence of CSFL was $32.3 \%$. The incidence of anterior decompression (AD), posterior decompression (PD), and CD (CD) were 20.5\%, 31.1\%, and $41.8 \%$, respectively. The incidence of CSFL from CD was much higher than that pf other surgical approaches. Takahata et al. (11) reported an incidence of CSFL after CD as high as $40 \%$. This was because the posterior approach of CD used direct decompression, which requires the removal of the ventral compression of the spinal cord from the rear. As a result, spinal cord occlusion led to a narrow space and limited visual field, which was the reason why the incidence of CSFL of circular decompression surgery was higher than that of other approaches. The surgical method in our study was CD, and the incidence of CSFL was $28.0 \%$, which was slightly lower than Hu's result. The improvement of surgical instruments is an important factor. During the operation, we used piezosurgery to assist in spinal cord decompression. However, even if the incidence of CSFL can be reduced through an operator's surgical techniques and improved surgical instruments, the high prevalence of CSFL among 
Table 2 Univariate analysis for predictors of postoperative CSFL

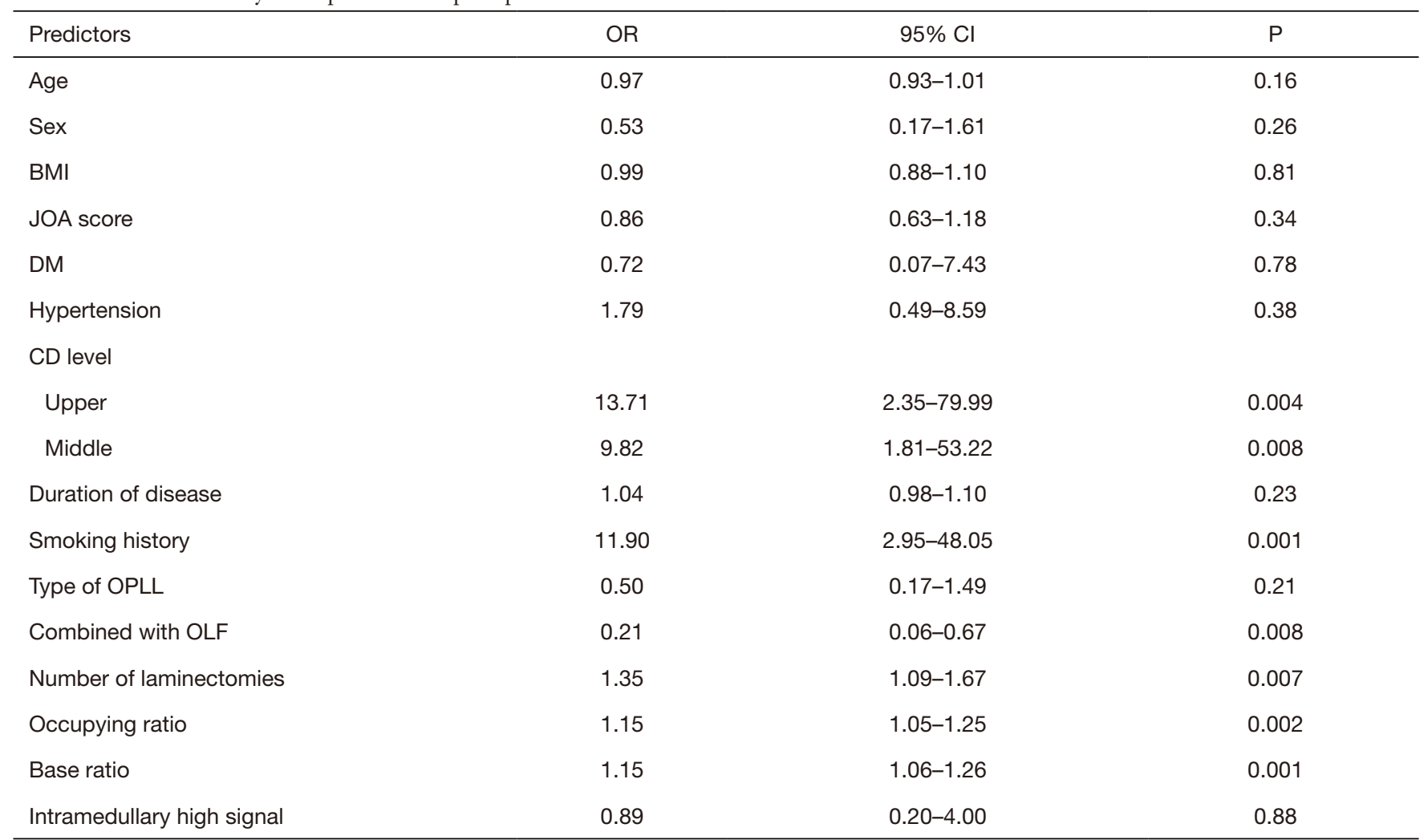

CSFL, cerebrospinal fluid leakage; BMI, body mass index; JOA, Japanese Orthopaedic Association; DM, diabetes mellitus; CD, circumferential decompression; OPLL, ossification of posterior longitudinal ligament; OLF, ossification of the ligamentum flavum.

Table 3 Multivariate analysis for predictors of postoperative CSFL

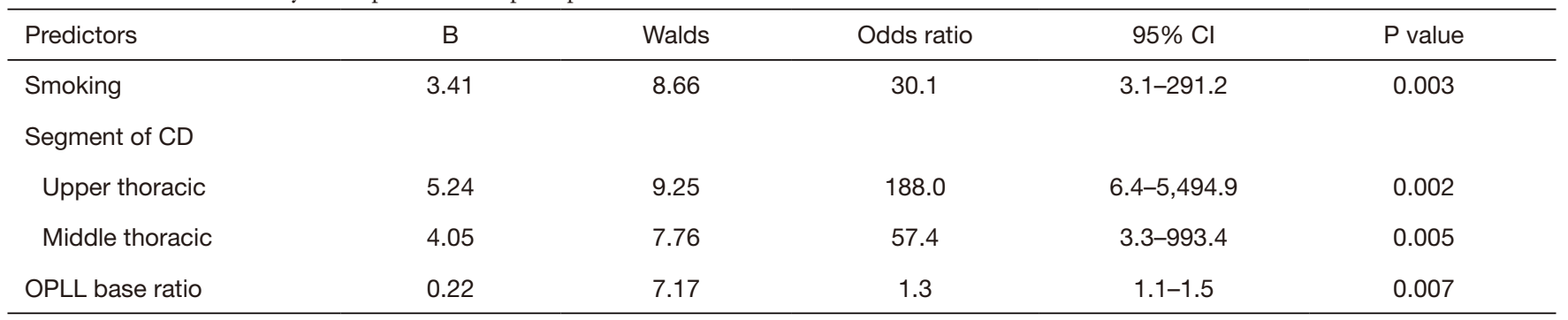

CSFL, cerebrospinal fluid leakage; CD, circumferential decompression; OPLL, ossification of posterior longitudinal ligament.

thoracic OPLL patients (a third of the cases in our study) still poses a great risk. Therefore, predicting the factors that can lead to CSFL before surgery and screening out OPLL patients who are at high-risk of developing CSFL through $\mathrm{CD}$ can be a helpful way for surgeons to preoperatively advise patients of the associated risks of $\mathrm{CD}$.

At present, there are few reports that explore predictors of CSFL after CD, and there are no universally acknowledged conclusions. Sun et al. (13) found that patients who underwent a laminectomy for more than three segments [odds ratio $(\mathrm{OR})=2.4 ; \mathrm{P}=0.01$ ] had a higher chance of CSFL occurring ( $\mathrm{OR}=2.4 ; \mathrm{P}<0.01)$. In this study, although the number of removed vertebral plates in the CSFL group was significantly higher than that in the nonCSFL group (5.9 vs. 3.7; $\mathrm{P}=0.01$ ), there was no statistical difference in the logistic regression analysis. This was 


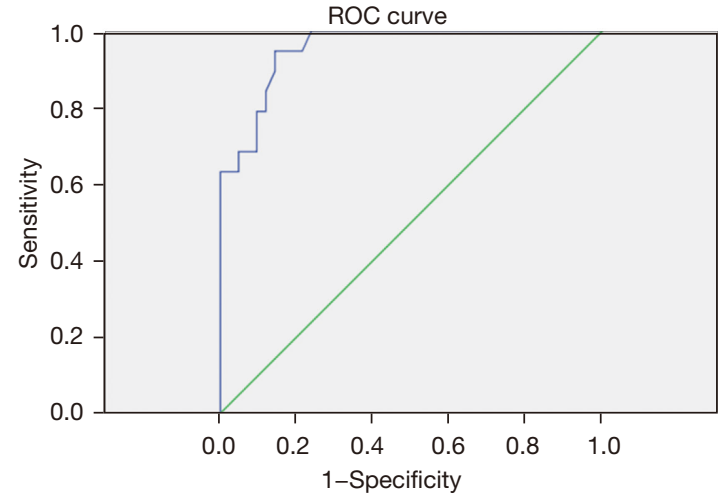

Figure 2 ROC curve showing the overall ability of the model to classify CSFL. The area under the curve $=0.955$. ROC, receiver operating characteristic; CSFL, cerebrospinal fluid leakage.

related to differences in the etiology and surgical methods of the two studies. For example, Sun et al. only studied cases with OLF, the surgical method was a posterior approach laminectomy and decompression, and the dural injury occurred only in the dorsal spinal cord. By contrast, in our study, the included patients had OPLL, the surgical method was $\mathrm{CD}$, and the dural injury occurred more often in the ventral spinal cord. As a result, the factors leading to CSFL were more complex. In a study of dural injury during lumbar surgery, Ahn et al. (14) found that patients with diabetes (OR $=2.3 ; \mathrm{P}=0.04)$ or a history of smoking $(\mathrm{OR}=3.4 ; \mathrm{P}=0.02)$ were more likely to develop CSFL. The smoking history of our own study was also found to be a risk factor for CSFL after $\mathrm{CD}(\mathrm{OR}=30.1 ; \mathrm{P}=0.003)$, which is consistent with the study by Ahn et al. It is widely believed that smoking can lead to thinning of the fascia and decreased toughness (15). The dura mater, though different from the abdominal pelvic fascia, has a similar composition and structure. It can therefore partly explain why smoking increases the risk of CSFL. Hu et al. (10) found that CSFL is more likely to occur in T5-7 circular decompression surgery than in other segments. In our study, the probability of CSFL occurring in the upper thoracic segment $(\mathrm{OR}=188.0 ; \mathrm{P}=0.002)$ and the middle thoracic segment $(\mathrm{OR}=57.4 ; \mathrm{P}=0.005)$ was also significantly higher than that of the lower thoracic segment. This is mainly because the middle and upper thoracic segments were narrower and presented a limited surgical space, so they were more likely to cause dural injury.

The shape of the OPLL itself may also be the cause of postoperative CSFL. The sagittal and transverse diameters of OPLL were used to evaluate the occupying ratio and OPLL base ratio. It was found that the vertebral occupying ratio and OPLL base ratio in the CSFL group were higher than those in the non-CSFL group. The multivariate regression analysis showed that the OPLL base ratio was a predictor of postoperative CSFL. The wider the OPLL base was, the more likely was postoperative CSFL occurrence (OR $=1.3, \mathrm{P}=0.007)$. Du et al. (16) came to a similar conclusion in studying the risk factors of CSFL after use of anterior approach cervical OPLL decompression. Du et al. collected the data of 90 patients who underwent anterior approach cervical decompression to treat cervical OPLL and found that CSFL is more likely to happen during resection of a widebased OPLL (OR $=1.09 ; \mathrm{P}=0.012$ ) than that of a narrowbased OPLL. The reason was that the base at both headand-tail of the OPLL was cut off in advance of removing the OPLL ossific block. As a result, the wide base of the OPLL increases the difficulty of resection, causing greater dural invasion and a higher chance of developing CSFL. After cutting the base at both the head-and-tail of the OPLL, we used the "collapse method" to push the free OPLL ossific block directly to the anterior vertebral gap without directly contacting the final part of the OPLL ossific block near the midline. Therefore, the OPLL sagittal diameter had little effect on the formation of CSFL, which was consistent with the results of regression analysis in this paper.

From our research, no literature currently exists regarding the predictive model of CSFL after thoracic CD. This study attempted to establish a predictive model for determining the probability of CSFL occurring after the CD of the thoracic OPLL. The logistic regression analysis found that smoking history, segment of CD, and the OPLL base ratio were statistically significant. They can therefore be used to predict the probability of postoperative CSFL occurring. In general, an area under the ROC curve $>0.6$ indicates that the model has good predictability. The ROC of the model was 0.955 , indicating that the model can predict the risk of CSFL after CD well.

Clinical relevance of the current study related to the identification of risk factors associated with CSFL. Firstly, we found that a history of smoking, the segment of CD in the upper or middle thoracic spine, and a wide base of OPLL were independent risk factors for postoperative CSFL. These results should be taken into considered during clinical decision-making. In addition, we suggested the implication of piezosurgery technique during OPLL resection. The safety and efficiency of piezosurgery in the removal of bone structures have been widely acknowledged in academic circles. Compared with the traditional bone 

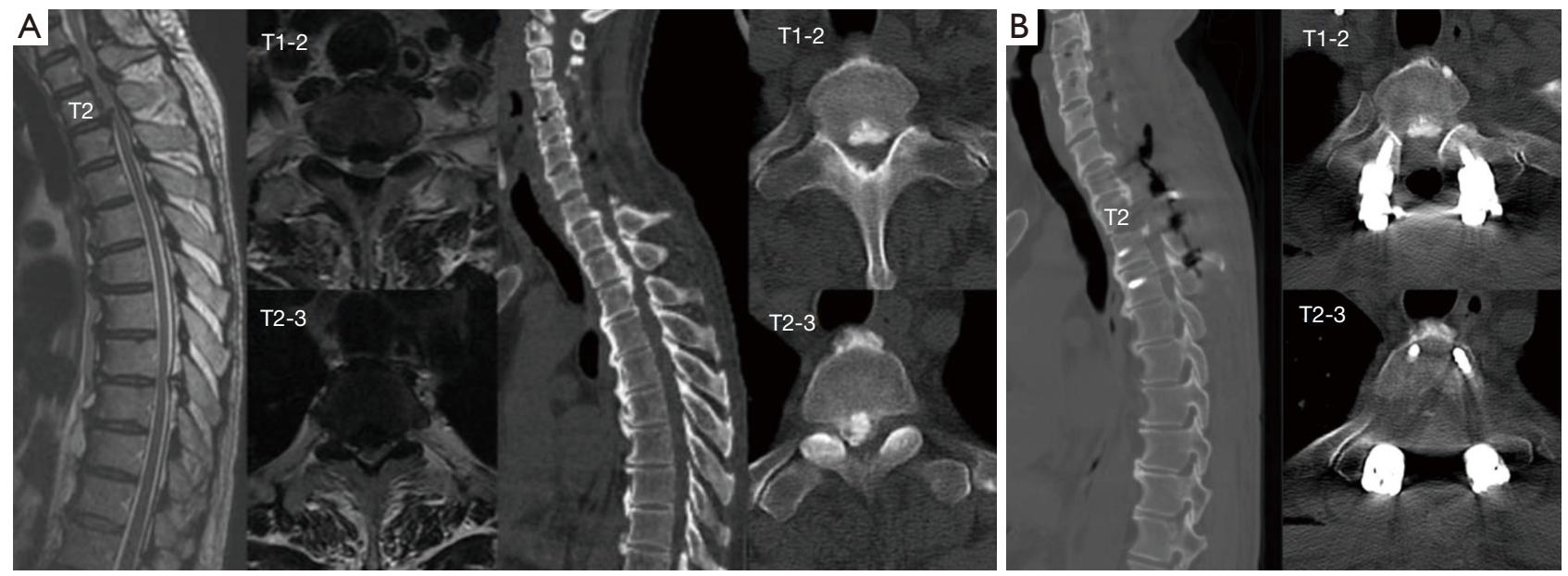

Figure 3 A typical case. A 60-year-old man presented with progressive exacerbation of lower limb weakness and walking instability for 6 months. The preoperative JOA score was 3. (A) The CT and MRI scans both showed continuous OPLL from T1 to T3 with the worst compression at T2-3; (B) the patient underwent T1-3 laminectomy and selectively T2-3 CD. Postoperative CT scans showed that OPLL was completely removed at T2-4, but was retained in the other regions. After a 1-year follow-up, the patient's JOA score was 9 and the recovery rate was $75.0 \%$. JOA, Japanese Orthopaedic Association; MRI, magnetic resonance imaging; OPLL, ossification of the posterior longitudinal ligament; $\mathrm{CD}$, circumferential decompression.

knife or high-speed drill, piezosurgery's high-energy, highfrequency, and low-amplitude characteristics can achieve basically no vibration when removing ossified blocks. The characteristics of "cutting soft and not cutting hard" also help to avoid dural injury. As a result, the rational use of piezosurgery can effectively reduce the occurrence of CSFL.

We should also mention some limitations to this study. First, as the study's aim was to explore the predictive model of CSFL, patient follow-up did not occur over a long period. Therefore, the long-term effects of CSFL on patients, such as pseudocyst formation, nerve function recovery, and so on, require further follow-up studies. Second, the number of patients who took part in the study was limited by the low incidence of thoracic OPLL and the limited necessity to perform CD. For future studies, a much larger number of patients should be recruited.

\section{Conclusions}

The predictive model established in this study had a high predictive effect. It shows that if patients with thoracic OPLL have a history of smoking, if the segment of CD is in the upper or middle thoracic spine, or if the OPLL has a wide base, there is a higher possibility for postoperative CSFL occurring. Using these factors, a surgeon can preoperatively warn patients of the probability of CSFL occurring after posterior transarticular osteotomy and CD.

\section{Acknowledgments}

Funding: None.

\section{Footnote}

Reporting Checklist: The authors have completed the STARD reporting checklist. Available at https://dx.doi. org/10.21037/apm-21-2323

Data Sharing Statement: Available at https://dx.doi. org/10.21037/apm-21-2323

Conflicts of Interest: All authors have completed the ICMJE uniform disclosure form (available at https://dx.doi. org/10.21037/apm-21-2323). The authors have no conflicts of interest to declare.

Ethical Statement: The authors are accountable for all aspects of the work in ensuring that questions related to the accuracy or integrity of any part of the work are appropriately investigated and resolved. All procedures performed in this study involving human participants were in accordance with the Declaration of Helsinki (as revised 
in 2013). The study was approved by ethics board of Peking University International Hospital [No. 2021-006(BMR)] and informed consent was taken from all the patients.

Open Access Statement: This is an Open Access article distributed in accordance with the Creative Commons Attribution-NonCommercial-NoDerivs 4.0 International License (CC BY-NC-ND 4.0), which permits the noncommercial replication and distribution of the article with the strict proviso that no changes or edits are made and the original work is properly cited (including links to both the formal publication through the relevant DOI and the license). See: https://creativecommons.org/licenses/by-nc-nd/4.0/.

\section{References}

1. Matsumoto M, Chiba K, Toyama Y, et al. Surgical results and related factors for ossification of posterior longitudinal ligament of the thoracic spine: a multiinstitutional retrospective study. Spine (Phila Pa 1976) 2008;33:1034-41.

2. Ma X, An HS, Zhang $Y$, et al. A radical procedure of circumferential spinal cord decompression through a modified posterior approach for thoracic myelopathy caused by severely impinging anterior ossification. Spine J 2014;14:651-8.

3. Xu ZW, Hu YC, Sun CG, et al. Treatment for Thoracic Ossification of Posterior Longitudinal Ligament with Posterior Circumferential Decompression. Orthop Surg 2017;9:206-14.

4. Yamazaki M, Mochizuki M, Ikeda Y, et al. Clinical results of surgery for thoracic myelopathy caused by ossification of the posterior longitudinal ligament: operative indication of posterior decompression with instrumented fusion. Spine (Phila Pa 1976) 2006;31:1452-60.

5. Li M, Meng H, Du J, et al. Management of thoracic myelopathy caused by ossification of the posterior longitudinal ligament combined with ossification of the ligamentum flavum-a retrospective study. Spine J 2012;12:1093-102.

6. Khan MH, Rihn J, Steele G, et al. Postoperative management protocol for incidental dural tears during degenerative lumbar spine surgery: a review of 3,183 consecutive degenerative lumbar cases. Spine (Phila $\mathrm{Pa}$ 1976) 2006;31:2609-13.

7. Hannallah D, Lee J, Khan M, et al. Cerebrospinal fluid leaks following cervical spine surgery. J Bone Joint Surg Am 2008;90:1101-5.
8. Galarza M, Gazzeri R, Alfaro R, et al. Evaluation and management of small dural tears in primary lumbar spinal decompression and discectomy surgery. J Clin Neurosci 2018;50:177-82.

9. Kawahara N, Tomita K, Murakami H, et al. Circumspinal decompression with dekyphosis stabilization for thoracic myelopathy due to ossification of the posterior longitudinal ligament. Spine (Phila Pa 1976) 2008;33:39-46.

10. Hu P, Yu M, Liu X, et al. A circumferential decompression-based surgical strategy for multilevel ossification of thoracic posterior longitudinal ligament. Spine J 2015;15:2484-92.

11. Takahata $M$, Ito $M$, Abumi $K$, et al. Clinical results and complications of circumferential spinal cord decompression through a single posterior approach for thoracic myelopathy caused by ossification of posterior longitudinal ligament. Spine (Phila Pa 1976) 2008;33:1199-208.

12. Hu P, Yu M, Liu X, et al. Cerebrospinal Fluid Leakage after Surgeries on the Thoracic Spine: A Review of 362 Cases. Asian Spine J 2016;10:472-9.

13. Sun X, Sun C, Liu X, et al. The frequency and treatment of dural tears and cerebrospinal fluid leakage in 266 patients with thoracic myelopathy caused by ossification of the ligamentum flavum. Spine (Phila Pa 1976) 2012;37:E702-7.

14. Ahn N, Ahn U, Post Z, et al. 39. Smoking, diabetes and excessive preoperative epidural steroid administration are risk factors for intraoperative dural tears. The Spine Journal 2004;4. DOI: https://doi.org/10.1016/ j.spinee.2004.05.040.

15. Goyal DKC, Divi SN, Bowles DR, et al. How Does Smoking Influence Patient-reported Outcomes in Patients After Lumbar Fusion? Clin Spine Surg 2021;34:E45-50.

16. Du YQ, Duan WR, Chen Z, et al. Risk Factors and Management of Dural Defects in Anterior Surgery for Cervical Ossification of the Posterior Longitudinal Ligament. World Neurosurg 2018;111:e527-38.

(English Language Editors: J. Goetz and J. Gray)

Cite this article as: Zhong J, Wen B, Chen Z. Predicting cerebrospinal fluid leakage prior to posterior circumferential decompression for the ossification of the posterior longitudinal ligament in the thoracic spine. Ann Palliat Med 2021;10(10):10450-10458. doi: 10.21037/apm-21-2323 